\title{
Comment on the article-“'Acetabular cup migration after primary cementless total hip arthroplasty in rheumatoid arthritis and its influencing factors: a comparative study with osteoarthritic hip"
}

\author{
Mohak Kataria $^{1}$ - Prasoon Kumar ${ }^{1} \cdot$ Vishal Kumar $^{1}$ \\ Received: 3 October 2020 / Accepted: 9 October 2020 / Published online: 20 October 2020 \\ (C) SICOT aisbl 2020
}

Dear Editor-in-chief,

We read the article on "Acetabular cup migration after primary cementless total hip arthroplasty in rheumatoid arthritis and its influencing factors: a comparative study with osteoarthritic hip" by Jun-Ki Moon et al. [1] and congratulate the authors on their well-thought retrospective analysis. We had an in-depth discussion of this article in our departmental journal club and would like to present forth certain queries and concerns.

1. In your study, you have attributed the increased total and proximal migration of the acetabular component in RA patients to their poor bone stock. Also, in the study by Kengo Takahashi et al. [2], it has been mentioned that low body mass index (BMI) is a significant risk factor for lower bone mineral density (BMD). However, in your methodology, the two groups (RA and OA) were matched for both BMD and BMI. Does this not nullify the effect of poor bone stock?

2. There is no mention of either the intra-operative stability of the acetabular cup or the number of screws used in each group and whether they were comparable.

3. We could not find any standard literature on the criteria of cup loosening as mentioned in your study.

Mohak Kataria

Kataria.mohak@gmail.com

1 Post Graduate Institute of Medical Education and Research, Sector 12, Chandigarh 160012, India
4. Acetabular cup loosening occurred in three cups in the RA group and in one cup in the OA group, showing no significant difference. So what exactly is the significance of cup migration that did not have any clinical implications [3]?

\section{References}

1. Moon J-K, Jung J-W, Kim Y, Yang J-H, Park Y-S, Kim Y-H (2020) Acetabular cup migration after primary cementless total hip arthroplasty in rheumatoid arthritis and its influencing factors: a comparative study with osteoarthritic hip. Int Orthop 44(6):1047-1053. https://doi.org/10.1007/s00264-020-04502-3

2. Takahashi K, Setoguchi T, Twaratsumida H et al (2015) Risk of low bone mineral density in patients with rheumatoid arthritis treated with biologics. BMC Musculoskelet Disord 16:269. https://doi.org/ 10.1186/s12891-015-0732-x

3. Krismer M, Stöckl B, Fischer M, Bauer R, Mayrhofer P, Ogon M (1996) Early migration predicts late aseptic failure of hip sockets. J Bone Joint Surg (Br) 78:422-426. https://doi.org/10.1007/s00264020-04502-3

Publisher's note Springer Nature remains neutral with regard to jurisdictional claims in published maps and institutional affiliations. 